\title{
Design and Analysis of OFDM System Employed in 5G MIMO Wireless Communication by using NI Hardware: USRP and Lab VIEW Software
}

\author{
Rashmi Yadav \\ Department of Electronics \& \\ Communication Engineering \\ KIET Group of Institutions \\ Ghaziabad, U.P, India
}

\author{
Vibhav Kumar Sachan, PhD \\ Department of Electronics \& \\ Communication Engineering \\ KIET Group of Institutions \\ Ghaziabad, U.P, India
}

\author{
Parvin Kumar \\ Department of Electronics \& \\ Communication Engineering \\ KIET Group of Institutions \\ Ghaziabad, U.P, India
}

\begin{abstract}
Orthogonal Frequency Division Multiplexing (OFDM) has turned into the adjustment procedure for some remote communication models. In a remote system, a flag transmitted into channel ricochets off from different surfaces bringing about multiple postponed forms of the transmitted flag landing at the collector. The multiple signs are gotten because of the reflections from huge articles, diffraction of electromagnetic waves around objects. This makes the got flag be contorted. OFDM gives resilience to such frequency particular channels and gives high information rates. In this paper we propose to examine the hypothesis of OFDM, reproduce the OFDM handset utilizing Lab VIEW and perform FFT examination.
\end{abstract}

Keywords

OFDM, FFT-OFDM, 1G, 2G, 3G, 4G ,5G \&Lab VIEW etc.

\section{INTRODUCTION}

Development of Mobile Communication Network

The mobile communication advancements are isolated into ages; a short review of these ages is given under below:

\section{$1.11 G$}

The original mobile communication which was presented in 1980s depended on the simple system. The most well-known simple 1G system was advanced mobile phone system (AMPS) which was propelled in United States. Nordic mobile telephone (NMT), total access communication system (TACS) and some other simple systems were likewise presented in 1980s over the Europe. The greater part of the models in 1G utilizes frequency adjustment strategies for voice signals. The range inside cell was separated into number of channels which was not effective regarding the accessible radio range, and this set a constraint on the quantity of calls that could be set aside a few minutes. Simple systems depended on circuit exchanging innovation and offers just voice communication and no information communication. After the presentation of $1 \mathrm{G}$ innovation mobile communications have experienced noteworthy changes and experienced gigantic development and the quantity of supporter came to about 20 million by 1990 .

\subsubsection{Key features and facilities of $1 G$ \\ - Based on analog system \\ - It supports data speed of up to $2.4 \mathrm{kbps}$ \\ - Cordless telephone}

\subsubsection{Essential disadvantages of $1 G$}

- Only voice, no information communication

- Low limit

- Poor handoff

- Less secure

- Poor voice connect

\section{$1.22 \mathrm{G}$}

A change to $1 \mathrm{G}$ communication appeared as $2 \mathrm{G}$ innovation which was presented in late 1980's and depended on low-band computerized information flagging. In this simple innovation was supplanted by Digital Access strategies, for example, TDMA (Time division multiple access) and CDMA (code division multiple access). The most mainstream $2 \mathrm{G}$ remote innovation is known as Global Systems for Mobile Communications (GSM). Global System for Mobile Communications, or GSM, utilizes TDMA innovation to help multiple clients. The TDMA separates information transmission, for example, a phone discussion, into sections and transmits each part in a short burst, doling out each piece a time space. With a PDA, the guest does not identify this discontinuity. Amid advancement over 20 years, GSM innovation has been ceaselessly enhanced to offer better administrations to its clients. GSM underpins 450 million cell supporters, with international wandering in around 140 nations and 400 networks.

CDMA utilizes spread range innovation to separate discourse into little, digitized portions and encodes them to distinguish each call. The CDMA recognizes multiple transmissions conveyed all the while on a solitary remote flag. It conveys the transmissions on that flag, liberating network space for the remote transporter and giving without interference calls to the client. The CDMA separates approaches a flag by codes, though TDMA separates them by time. The outcome in the two cases is an expanded network limit with respect to the remote transporter and an absence of interference for the visitor.

1.2.1 Key features and facilities of $2 G$

- Enhanced Spectrum productivity

- Provides information rate of up to $64 \mathrm{kbps}$

- Improved system limit, and network scope

- Roaming office

- Voice and information administrations

- Enhanced security 


\subsubsection{Basic disadvantages of $2 G$}

- It doesn't bolster high information rates.

- Weaker advanced flag

- Unable to deal with complex information

\section{$1.33 \mathrm{G}$}

The 3G acquired incredible transformation mobile communication world. The 3G satisfies the determinations of International Mobile Telecommunications-2000 (IMT-2000), the official International Telecommunication Union which proposed to give remote access to global telecommunication system. To meet the IMT-2000 principles, a system is required to give top information rates of no less than 200 $\mathrm{kbit} / \mathrm{s}$. The most critical IMT-2000 recommendations are the Universal Mobile Telecommunications System (UMTS) as the successor to GSM. The UMTS utilizes the W-CDMA, TD-CDMA, or TD-SCDMA air interfaces in which WCDMA is the most prominent air-interface innovation for the UMTS. The primary segments incorporate BS (Base Station) or gesture B, RNC (Radio Network Controller), aside from WMSC (Wideband CDMA Mobile Switching Center) and SGSN/GGSN

The W-CDMA gives extra points of interest of high exchange rate, and expanded system limit and communication quality by factual multiplexing. The WCDMA uses proficiently the radio range, in light of the fact that the CDMA system empowers every single base station to utilize a similar frequency. In the WCDMA system, the information is part into isolated bundles, which are then transmitted utilizing parcel exchanging innovation, and the bundles are reassembled in the right grouping at the beneficiary end by utilizing the code that is sent with every bundle. The UMTS systems are intended to give a scope of information rates, contingent upon the client's conditions, giving up to $144 \mathrm{kbps}$ to moving vehicles, up to $384 \mathrm{kbps}$ for walkers and up to 2 Mbps for indoor or stationary clients. The $3 \mathrm{G}$ fundamentally centered around multimedia applications, for example, video calling, videoconferencing for mobile phones, enhanced limit, world wandering, minimal effort, better similarity, fast information.

\subsubsection{Key highlights and offices of $3 G$}

- Faster information rates

- Supports multimedia applications, for example, video and photography

- Value included administrations like mobile TV, GPS (global

situating system), video call and video conferencing.

- High speed mobile internet access

- Increased limit

\subsubsection{Fundamental hindrances of $3 G$}

- Requires $3 \mathrm{G}$ perfect handsets

- The cost of moving up to $3 \mathrm{G}$ gadgets is costly

- Power utilization is high

- $3 \mathrm{G}$ requires nearer base stations which is costly.

\subsection{G}

The $4 \mathrm{G}$ is a developing innovation in the field of communication. As the information prerequisites expanded, efforts were made to enhance the downlink and uplink throughput rates by utilizing higher tweak systems. Third Generation Partnership Project (3GPP) propelled the Long Term Evolution (LTE) venture in November 2004 with a specific end goal to guarantee the proceeded with aggressiveness of the UMTS later on. As LTE is considered as the development of all inclusive mobile telephone system (UMTS), consequently LTE's comparable segments are along these lines named advanced UMTS earthly radio access
(EUTRA) and advanced UMTS earthbound radio access network (EUTRAN). The essential engineering of LTE contains a different IP availability layer for all the IP based administrations and Evolved Packet System (EPS) which handles the general communication strategy. LTE is totally an all IP based system. Since there are arrangements in LTE for inter-operation with existing systems, there are different ways accessible to interface with LTE. An administrator with a GPRS/EDGE network or Non-3GPP systems can associate with a LTE network. Because of this expanded adaptability, LTE is the decision of dominant part of operators around the world. By utilizing Orthogonal Frequency Division Multiple Access (OFDMA), LTE will have the capacity to give download rates of around $100 \mathrm{Mbps}$ for multi-reception apparatus (2x2), multiple-input multiple yield (MIMO) for the most elevated class terminals. For these terminals transfer rate is around $50 \mathrm{Mbps}$. In addition, it gives better portability, proficient radio use, abnormal state of security, adaptable range use, decreased deferral/inertness, cost effective sending and different favorable circumstances which make LTE more solid and easy to use.

\subsubsection{Key highlights and offices of $4 G$}

- High otherworldly productivity

- High voice quality

- Easily access internet, spilling media, video calling and so on

- Very low dormancy

- Simple convention design

- Efficient multicast/communicate

\subsubsection{Essential burdens of $4 G$}

- Higher information costs for customers

- It is exceptionally costly and difficult to actualize

- Complex hardware

- Power use is more.

\section{Evoluation of Mobile Communication Network}

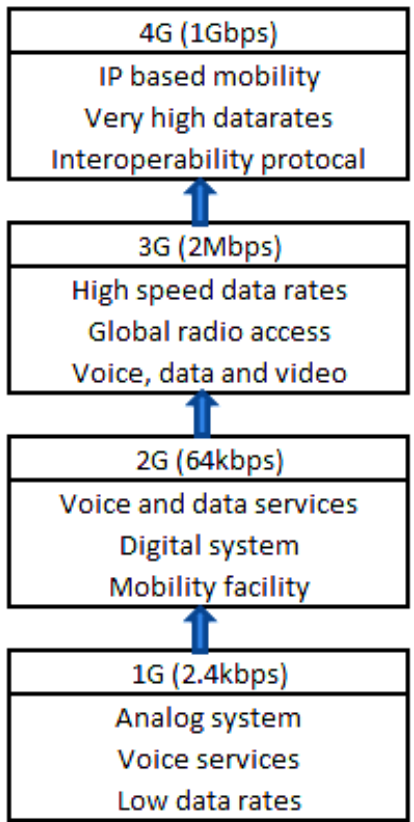

Fig 1: Evolution of mobile communication network

\section{$1.55 \mathrm{G}$}

As the fifth era of remote innovation, known as $5 \mathrm{G}$, is relied upon to make the "network of networks"— which will convey higher data transmission and lower dormancy than at any 
other time-mobile network operators (MNOs) should develop in three essential ways:

\subsubsection{Densification}

Densification will be important to get network access nearer to singular clients-along city lanes, in structures and wherever in the middle. By including more radio wires, little cell locales and in addition moving up to MIMO and area part innovations, MNOs will be on track to offer velocities which should be no less than 10 times faster than the current $4 \mathrm{G}$ innovation.

\subsubsection{Virtualization}

To adequately oversee range, MNOs should move crafted by physical hardware to virtualized conditions working in unified server farms utilizing arrangements, for example, incorporated radio access networks (C-RAN), network work virtualization (NFV) and cell virtualization.

\subsubsection{Optimization}

In the event that the Internet of Things (IoT) will be 20 billion in number by 2020, MNOs should plan their networks to oblige the extensive request by looking for more effective network arrangements, for example, drawing their processing assets nearer to the edge.

With the vision of network innovation proceeding to develop, Communication Scope's understanding and concentrate on advance can enable you to open the power and capability of $5 \mathrm{G}$.

\section{OFDM}

The interest for fast remote applications and restricted RF signals data transfer capacity is expanding step by step. New applications are rising in the wired systems, as well as in the remote mobile systems. At present, just low rate information administrations are accessible for mobile applications. Be that as it may, there is an interest for high information rates for multimedia applications. In single bearer system, the symbol span decreases with an expansion in information rate and therefore the impact of Inter symbol Interference (ISI) turns out to be more extreme. ISI, in remote communication systems, is created because of the memory of dispersive remote channels [1]. When in doubt, the impact of ISI on mistake performance of the system is irrelevant, as long as the defer spread is fundamentally shorter than the length of one transmitted symbol [2]. This infers the symbol rate bolstered by the communication system is essentially constrained by ISI. In the event that the information rate surpasses the furthest reaches of information transmission over the channel, an instrument must be executed with a specific end goal to battle the impacts of ISI. Channel leveling strategies can be utilized to smother the echoes caused by the channel. Be that as it may, such equalizers posture troubles continuously systems working at a few Mbps speed with reduced, ease hardware. Multicarrier regulation strategies act the hero in such circumstances. Multicarrier adjustment, and particularly OFDM, is one of the promising hopefuls [3] that utilize an arrangement of subcarriers keeping in mind the end goal to transmit the information symbols in parallel over the communication channel. It enables the communication system to transmit the information at a lower rate on multiple subcarriers and the throughput of multicarrier system stays equivalent to the single transporter system. This enables us to outline a system supporting high information rates, while keeping up symbol lengths any longer than the channel postpone spread, consequently improving the requirement for complex channel evening out component and can without much of a stretch battle the impact of ISI [2], [4].

\subsection{OFDM WORKING PRINCIPLE}
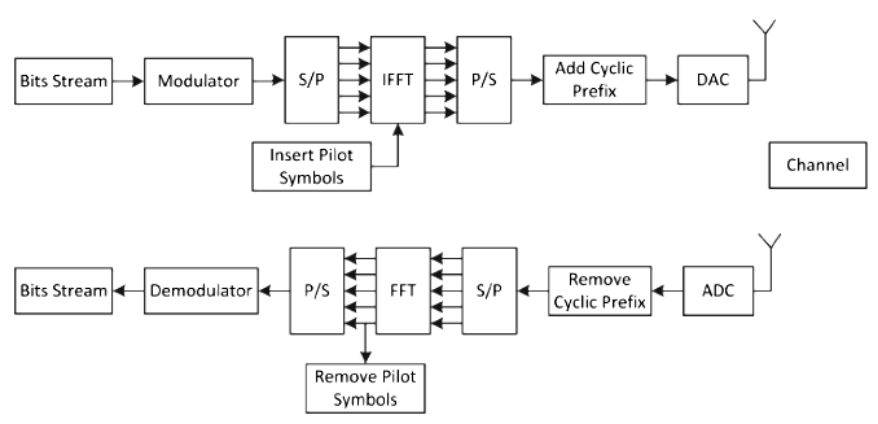

Fig 2: Working Principle of OFDM System

As appeared in above figure, first the transmitted information is carefully balanced utilizing adjustment plans. Generally QAM and PSK advanced tweak plans are utilized to regulate the transmitted flag. The yield of the modulator is changed over in to parallel flag. By this system flag is transmitted through subcarriers which are orthogonal to each other Due to orthogonally property, sub channels are not covered to each other, and ICI (Inter channel Interference) issue is diminished. The yield of the serial to parallel converter is then connected to IFFT (Inverse Fast Fourier Transform) .By utilizing IFFT the otherworldly portrayal of the information is moved in to time area, which is significantly more computationally proficient. The Cyclic prefix plot is utilized at the yield of the IFFT [5].

\subsection{SIMULATION MODEL}

\section{FFT based OFDM system model}

Orthogonal Frequency Division Multiplexing (OFDM) plot depends on the multicarrier communications system. In OFDM the spectra of subcarriers cover on each other however the frequency dispersing between them is chosen with the end goal that the subcarriers are numerically orthogonal to each other. Fig.3 demonstrates a piece outline of a fundamental OFDM system. Discrete Fourier Transform (DFT) effectively executed by Fast Fourier Transform (FFT) is utilized to balance and demodulate the information groups of stars on the orthogonal sub-transporters. This baseband flag handling calculation adequately replaces the bank of I/Q modulators and demodulators that would somehow or another be required [6]. An OFDM trans-collector is appeared in Fig.2 the opposite transform square can either be IDWT/IFFT or forward transform piece can be DWT/FFT. The information generator utilized creates a bit stream. It is handled utilizing QPSK or M-ary QAM modulator to delineate information into symbols. These symbols are currently sent through IFFT square to perform IFFT operation to create $\mathrm{N}$ parallel information streams. Its yield in discrete time area is given by,

$$
X_{k(n)}=\frac{1}{\sqrt{\mathrm{N}}} \sum_{i=0}^{N-1} \mathrm{X}_{\mathrm{m}}(\mathrm{i}) \mathrm{e}^{\left(\frac{\mathrm{j} 2 \pi \mathrm{ni}}{\mathrm{N}}\right)}
$$

The transformed yield is presently affixed with cyclic prefix. The cyclic prefix (CP) is included before transmission, to alleviate ISI impact. It is typically $25 \%$ of the last piece of the first OFDM symbol and this information is gone through AWGN channel with legitimate info control set. At the 
recipient, the invert operation is done to acquire the first information back. The CP is expelled and handled in the FFT piece lastly went through demodulator for information recuperation. The yield of the FFT in frequency area is offered by, to create a baseband OFDM symbol, a serial digitized information stream is first channel coded and afterward tweaked utilizing stage move keying (PSK) or quadrature adequacy balance (QAM). These information symbols are changed over from serial-to-parallel into $\mathrm{N}$ information group of stars focuses before balancing the subcarriers utilizing IFFT, where $\mathrm{N}$ is the quantity of IFFT focuses. The time space OFDM regulated symbol yield of the IFFT is changed over back to a serial stream and a protect interval as cyclic prefix is added to each OFDM symbol. The essential heartbeat shape for the symbols is rectangular which have substantial data transmission because of its sinc formed range. Accordingly windowing is important to lessen the out of band vitality of the side projections. At that point the symbol stream is changed over to simple form for pass-band handling and transmission. The collector performs the correct inverse of the transmitter. After pass-band preparing at the RF front-end, the baseband handling will be performed.

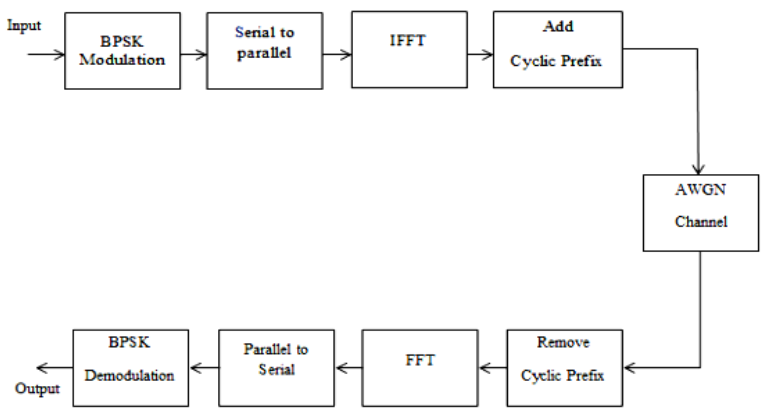

Fig 3: FFT-Based OFDM System

This incorporates protect interval evacuation and serial-toparallel change before the FFT demodulation. Besides, synchronization and channel estimation are improved the situation interference free subcarrier demodulation utilizing FFT and furthermore group of stars deciphering [7].

\section{OFDM IMPLEMENTATION}

In the fig. 4 shows the working setup of OFDM system implementation using LabVIEW software and USRP hardware.

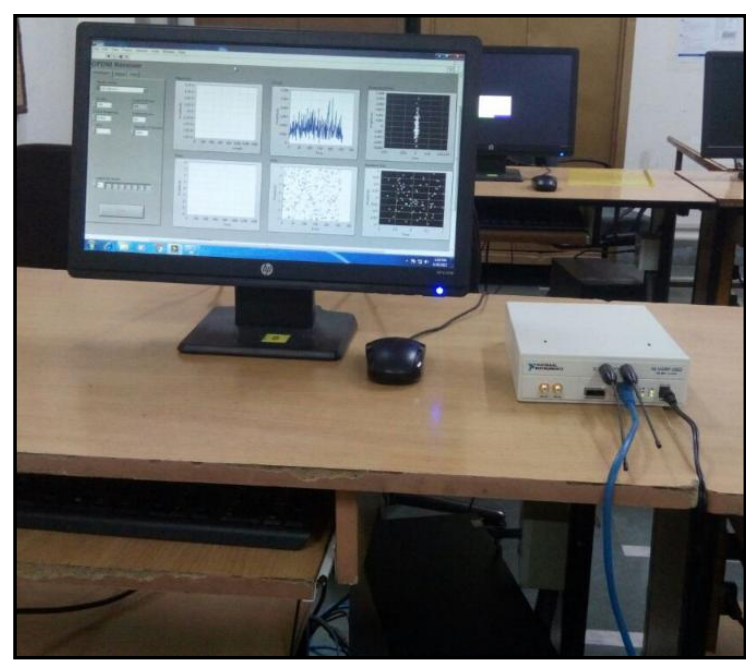

Fig 4: Working Setup

\section{RESULT AND DISCUSSION}

The USRP Hardware DriverTM (UHD) software API supports application development on all USRP SDR products. Using a common software interface is critical as it increases code portability, allowing applications to transition seamlessly to other USRP SDR platforms when development requirements expand or new platforms are available. Hence, it enables a significant reduction in development effort by allowing you to preserve and reuse your legacy code so you can focus on new algorithms. For more detailed information please visit our knowledge based article on UHD.

UHD also offers cross-platform support for multiple industry standard development environments and frameworks, including RFNoC, GNU Radio, and LabVIEW and Mat lab/Simulink. And to ensure you have no restrictions on how you use UHD, it is available on Linux, Windows, and Mac OS.

In fig 5.We have shown OFDM transmitter parameters OFDM design implements a complete parameter with device name 192.168.10.3, 1Q rate $=5 \mathrm{M}$, coerced IQ rate $=5 \mathrm{M}$, carrier frequency $=910 \mathrm{M}$, active antenna $=\mathrm{TX} 1$, gain $=9$, number of bits $=1250$ and outgoing size $=1600$ over FFT channel bandwidth. This combination of parameters makes for an ideal baseline waveform to experiment with, as it does not impose too many constraints on the RF section.

IQ rate: I/Q data shows the variation in magnitude (or amplitude) and phase of a sine wave. If amplitude and phase changes happen in an order, defined way, we can use these amplitude and phase changes to generate information upon a sine wave, a process known as modulation

Coerced IQ rate returns the actual I/Q rate, in samples per second $(\mathrm{S} / \mathrm{s})$, for this session, coerced to the capabilities of the device

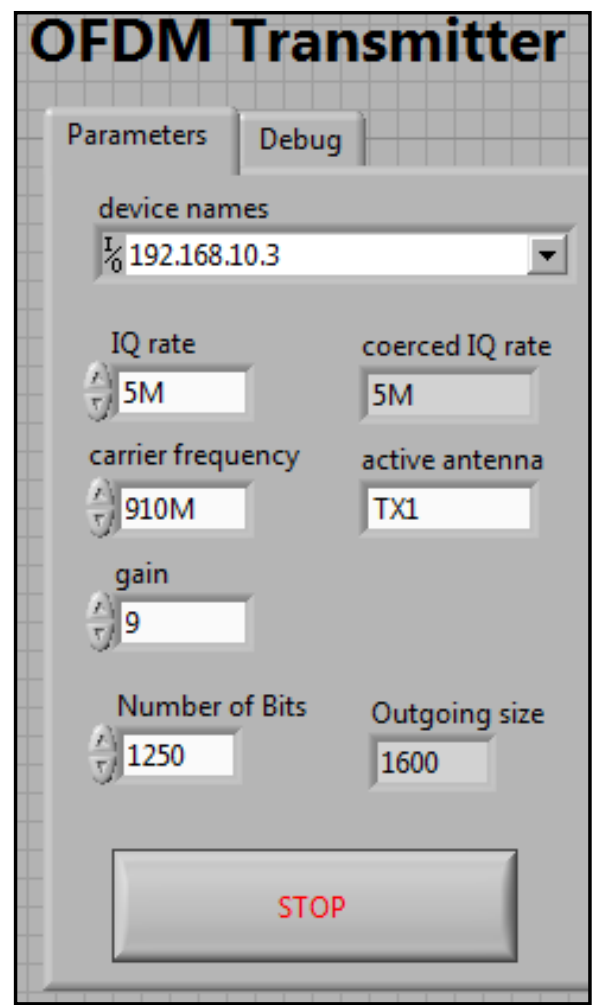

Fig 5: OFDM transmitter 
Fig 6 shows the Plotting of outgoing constellation between amplitude and time in OFDM transmitter.

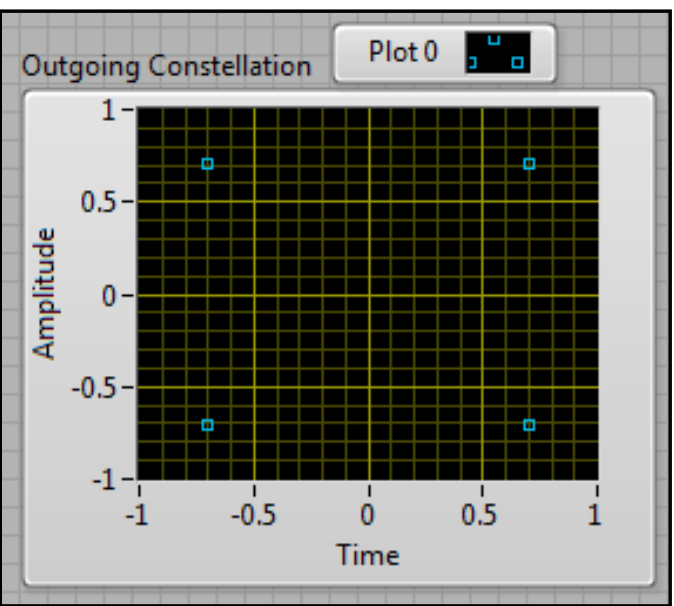

Fig 6: Outgoing constellation between amplitude and time

Fig 7 shows the FFT plotting of amplitude and time in OFDM transmitter.

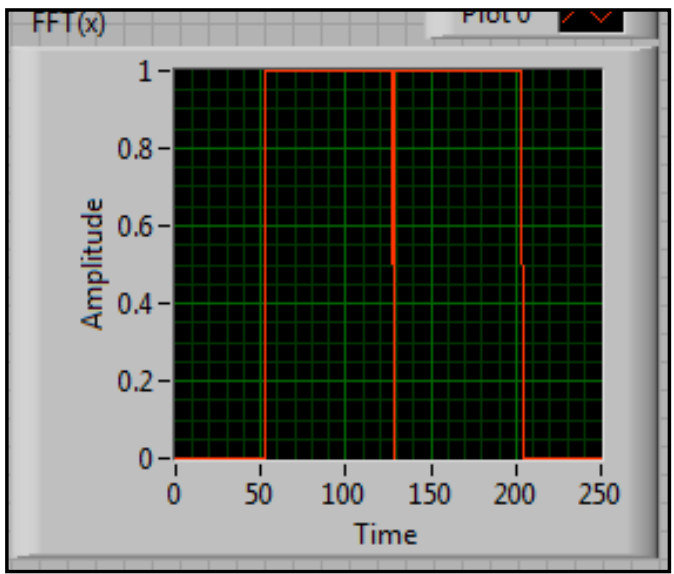

Fig 7: FFT plotting between amplitude and time

Fig 8 shows phase plotting of amplitude and time.

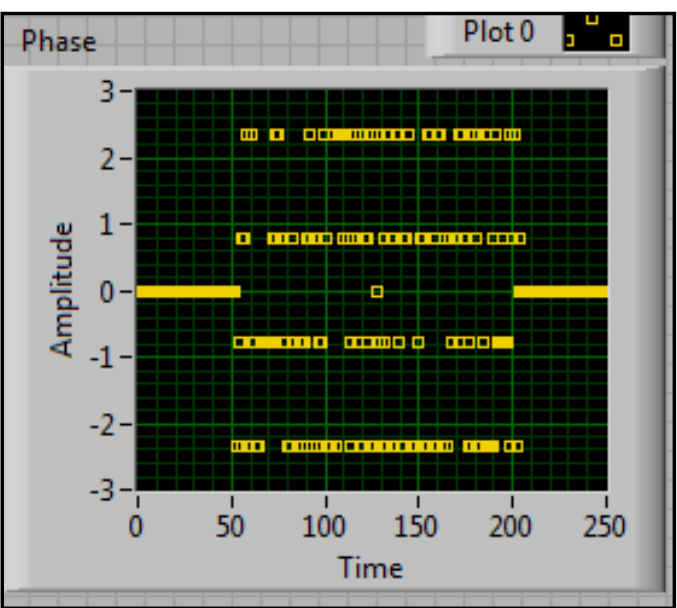

Fig: Phase plotting at transmitter

Fig 9 shows the receiver parameter in which we have taken IQ rate $5 \mathrm{M}$, coerced IQ rate $5 \mathrm{M}$, carrier frequency $910 \mathrm{M}$, active antenna RXI , gain 3 and number of samples 1500.

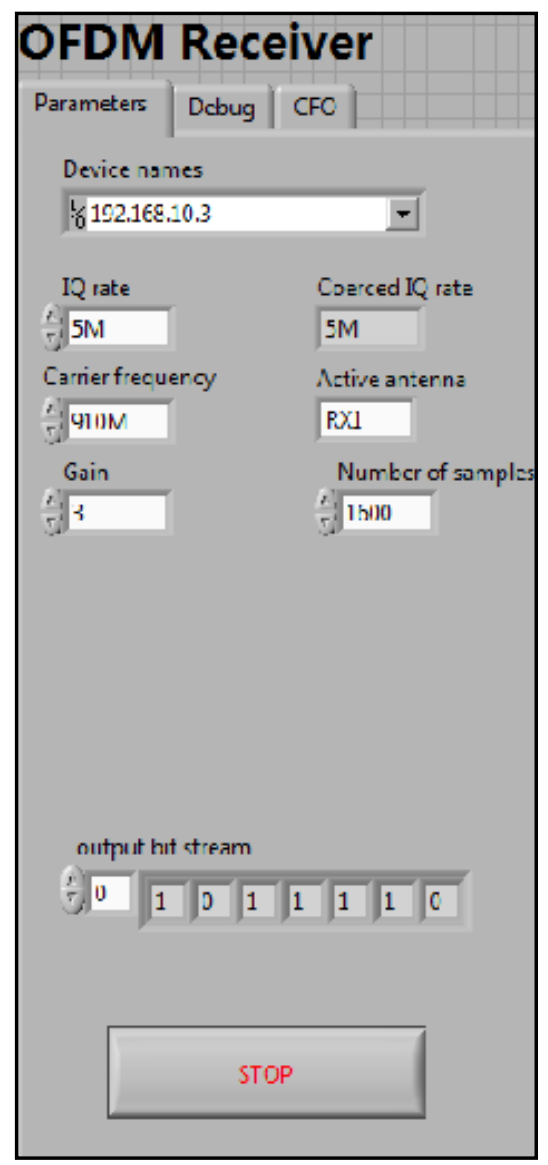

Fig 9: OFDM receiver

Fig 10 shows the FFT plotting between amplitude and time. Maximum amplitude is 0.005 at 130 .

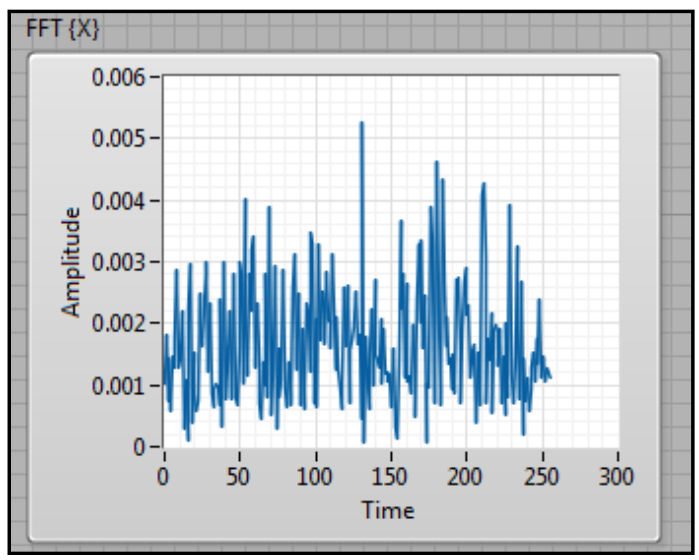

Fig 10: FFT plotting at receiver

Fig 11 shows the pattern of unequalized data between amplitude and time, which is not showing a exact point value. 


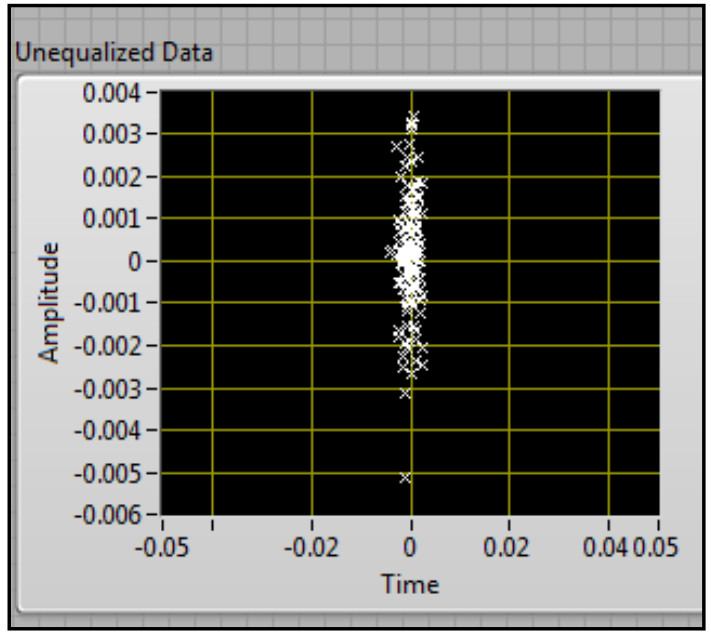

Fig 11: Unequalized data

Fig 12 shows the phase value graph between amplitude and time.

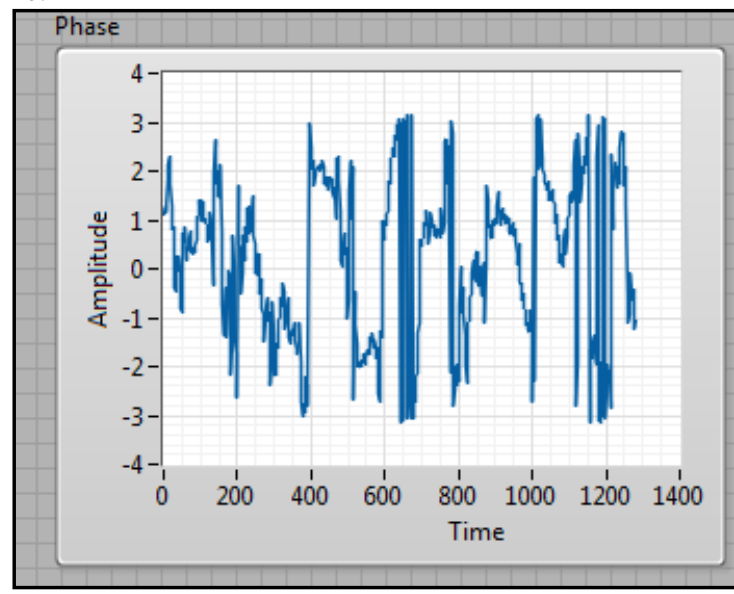

Fig 12: Phase plotting at receiver

Fig 13 shows the equalized data graph between amplitude and time, in equalized graph we can generate values at any point and we get exact value of sending QAM constellation points. And check amplitude at different values of time.

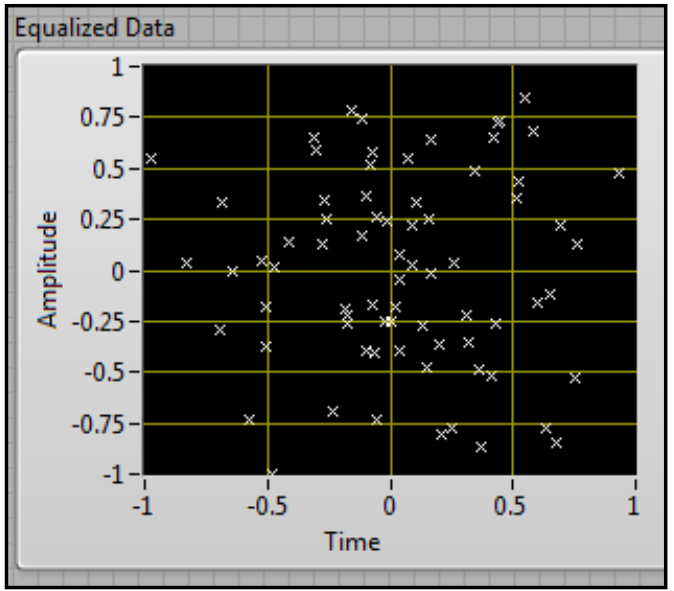

Fig 13: Equalized data at receiver

\section{CONCLUSION}

The demand for high data rate wireless communication has been increasing dramatically over the last decade. OFDM, which is the most emerging multi-carrier modulation technology of this era, can solve this problem significantly. This paper has provided an analysis of OFDM behaviors, principles and analyzed different techniques error reduction and frequency offset estimation that improve performance of OFDM for wireless communications. However, other literature shows that OFDM has a strong anti-multi path interference capability in a high-speed data transfer conditions and have also high spectral efficiency. The paper has also explored a brief overview of OFDM applications for wireless communications. Lastly the simulation results found with the help of working setup of OFDM system implementation using LabVIEW software and USRP hardware which conclude the successful transmission and reception of information bit using 4 QAM techniques with equalized data and amplitude after OFDM receiver.

\section{FUTURE WORK}

Wireless communication system is a vast field to work on and the number of generations being developed one by one and some of the improved functions in them, in each subsequent generation that led to the growth and development of society. Returning to $4 \mathrm{G}$, he provides the usual voice and the transfer of other services on the $3 \mathrm{G}$ network, and then includes a broadband service along with any other service as development in $4 \mathrm{G}$. Then $5 \mathrm{G}$ is completely independent technology and does not define the number of services it will provide in the future.

The finding of this thesis will provide a methodology to design OFDM system with LabVIEW software and USRP hardware. This is estimated that the various challenges to implement 5G technique will be meet out and the current and future requirement will be solved

\section{REFERENCES}

[1] Simon, M.K., Alouini M.S., Digital Communication over fading channels, 2nd edition, wiley, new york, 2005. [23] li y., stüber g.l., orthogonal frequency division multiplexing for wireless communications, springerverlag, 2006.

[2] Schulze h., luders c., theory and applications of ofdm and cdma: wideband wireless communications, wiley, 2005.

[3] Van nee r., prasad r., ofdm for wireless multimedia communications, artech house, 2003.

[4] Schulze h., luders c., theory and applications of ofdm and cdma: wideband wireless communications, wiley, 2005.

[5] Helmut bolcskei,eth zurich "mimo-ofdm wireless systems:basics, perspectives, and challenges" ieee wireless communication, august 2006.

[6] Mrs. Veena m. B. Dr. M. N. Shanmukhaswamy, "performance analysis of dwt based ofdm over fft based ofdm and implementing on fpga" international journal of vlsi design \& communication systems (vlsics) vol.2, no.3, september 2011.

[7] Swati sharma, sanjeevkumar, "ber performance evaluation of fft-ofdm and dwt-ofdm" international journal of network and mobile technologies issn 22299114 electronic version vol 2 / issue 2 / may 2011.

[8] S.gupta, parvin kumar kaushik and s.k sharma "a review on mobile technology evolution, rof and rofso system"2 $27^{\text {th }}$ sept 2015 . 